\title{
Propagation Directions of Ocean Surface Waves inside Tropical Cyclones
}

\author{
PAUL A. HWANG \\ Remote Sensing Division, Naval Research Laboratory, Washington, D.C. \\ EDWARD J. WALSH \\ Physical Sciences Division, NOAA/Earth System Research Laboratory, Boulder, Colorado
}

(Manuscript received 29 January 2018, in final form 30 April 2018)

\begin{abstract}
Surface wave propagation inside tropical cyclones (TCs) is complicated and multiple wave systems are frequently observed. The directional wave spectra acquired by hurricane hunters are analyzed to quantify its azimuthal and radial variations. Referenced to the hurricane heading, the dominate feature in the front half of the TC coverage area is single wave systems propagating toward left and left-front. Multiple wave systems are generally observed in the back and right quarters outside the radius of maximum wind (RMW). The directional differences and locations of occurrences of multisystem spectra are Gaussian distributed. The directional differences of the secondary and tertiary wave systems from the primary system are centered around $60^{\circ}-70^{\circ}$. The minor systems are more likely on the left-hand side of the primary system than on the right-hand side by a 3 -to- 1 ratio. The most likely azimuthal location of multisystem spectra is about $210^{\circ}$ counterclockwise from the heading. In the right-front quarter, waves propagate into the advancing wind field and experience extended airsea exchanges to grow higher and longer; in the left-rear quarter, they propagate away from the advancing wind field and are more likely younger seas. The radial variation of wave propagation is relatively minor except inside the RMW. A model describing the dominant wave propagation direction is presented. The regression statistics between modeled and measured wave directions show consistent agreement in 9 of the 11 datasets available for investigation. Causes for the significantly different statistics of the two remaining datasets include proximity to coast (a landfalling case) and rapid change in the hurricane translation speed or direction.
\end{abstract}

\section{Introduction}

National Oceanic and Atmospheric Administration (NOAA) hurricane reconnaissance and research missions combined active and passive microwave sensors to obtain simultaneous wind and wave measurements inside hurricanes (Wright et al. 2001; Walsh et al. 2002; Moon et al. 2003; Black et al. 2007; Fan et al. 2009b). For surface wave measurements, the NOAA WP-3D aircraft carried an airborne scanning radar altimeter (SRA) to obtain the 3D ocean surface topography, from which the $2 \mathrm{D}$ directional wavenumber spectrum was calculated by spectral analysis. Details of the measurement principle and processing procedure have been described in earlier publications (Walsh et al. 1985, 1989, 2002; Wright et al. 2001).

With precise spatial information of the measurement locations with respect to the hurricane center position,

Corresponding author: Dr. Paul A. Hwang, paul.hwang@nrl. navy.mil these simultaneous wind and wave data contain unprecedentedly detailed information on the radial and azimuthal variations of surface waves inside the hurricane coverage area (e.g., Wright et al. 2001; Walsh et al. 2002; Moon et al. 2003; Black et al. 2007; Fan et al. 2009b). For example, recent analyses of the hurricane hunter data produced a prototype of the fetch and duration model of tropical cyclone (TC) wind fields; the fetch and duration are defined at every location inside the hurricane coverage area (Hwang 2016; Hwang and Walsh 2016; Hwang and Fan 2017). Comparison of the directionally integrated 1D spectrum in the full field of the hurricane coverage area shows good agreement with published wind-wave spectrum models, and the systematic deviation between measured and modeled spectra is closely correlated to the wind and wave propagation directions, which show clear sinusoidal azimuthal variation and weak radial dependency (Hwang et al. 2017).

In this paper, we continue the analysis of the $2 \mathrm{D}$ surface wave spectra obtained by hurricane hunters with 
special emphasis on the propagation directions of the surface waves inside hurricanes. The information of the spatial variation of surface waves inside hurricanes is important for many applications, including hurricane remote sensing and air-sea exchanges. Particularly, it has been widely observed that long waves modify the wind speed dependency of the ocean surface roughness and its related properties such as the drag coefficient. Remote sensing of oceanographic parameters, for example, wind velocity and salinity, using active or passive microwave sensors relies on the knowledge of wind dependency of the ocean surface roughness, which is frequently the dominant limiting factor of the retrieval accuracy. The connection between the short-scale surface roughness components and long-scale waves remains a challenging subject of ongoing research. The high spatial resolution of the SRA 2D wave spectral measurements provides a great opportunity for a better understanding of the directional properties of surface wave propagation inside TCs.

Choosing 2 of the 11 available datasets with good spatial coverage ( 10 or more radial transects through the hurricane center), we first investigate the spatial patterns of wave directions by dividing the hurricane coverage area into eight azimuthal slices and four range divisions. The analysis gives a bird's-eye view of the wave propagation inside hurricanes. The result shows regions with distinctively mono- and multimodal directional distributions.

We then proceed to incorporate two more of the remaining datasets in developing a model for describing the dominant wave propagation direction. The model results are compared with measurements from all 11 missions, including the 4 used in model development. The regression statistics between modeled and measured wave directions show consistent agreement in 9 of the 11 datasets. The significantly different statistics of two datasets may be caused by proximity to coast (a landfalling case) and rapid change in the hurricane translation speed or direction.

The directional properties of multiwave spectra are described in statistical terms. The probability distribution functions (pdfs) are processed for the directional differences between the secondary-primary, tertiaryprimary, and tertiary-secondary systems, as well as the azimuthal phases where multiwave spectra occur. The pdfs are well represented by Gaussian distributions.

The comprehensive and precise spatial coverage of the hurricane hunter datasets far exceeds the level of spatial sampling density in previous analyses using composite in situ recordings from buoys or satellite chance passes. This improved spatial resolution allows us to examine the issue of wave propagation inside hurricanes without relying on human or model interpolation or extrapolation.

More details on previous analyses of wave propagation inside hurricane (e.g., Wright et al. 2001; Walsh et al. 2002; Moon et al. 2003; Young 2006; Fan et al. 2009a,b; Holthuijsen et al. 2012; Esquivel-Trava et al. 2015; Fan and Rogers 2016) were given in sections 3d and 3e of Hwang et al. (2017). Because of the limited amount of observations, studies using composite datasets from multiple TCs may have to make difficult compromises. For example, Hu and Chen (2011) combine hurricanes in different water depths without accounting for the bathymetry effects.

One reviewer had singled out the studies by Young (2006) and Collins et al. (2018). These two papers indeed provide the visual examples of the sparse coverage issue, and they had to rely heavily on numerical models to aid their analyses [see Fig. 4a of Young (2006) and Figs. 10 and 11 of Collins et al. (2018)]. The maximum wind speed in Collins et al.'s single-point measurements was $26 \mathrm{~m} \mathrm{~s}^{-1}$ (as stated in their abstract and illustrated in their Fig. 2; in other places they mentioned that there were five datasets with winds greater than $33 \mathrm{~m} \mathrm{~s}^{-1}$ ), so despite their title ("Directional wave spectra observed during intense tropical cyclones"), the study was in fact based primarily on numerical model results to extrapolate their field observations under tropical storms to tropical cyclone conditions.

In this study, we try to stay true to data and to decouple the field measurements from the "physics" or "dynamics" imposed by human or numerical models. Ultimately, we seek to advance beyond the general qualitative description of wave propagations in various quarters with a parametric model, established with measurements from hurricane reconnaissance and research missions, to provide quantitative information of the wave propagation direction at every location inside the hurricane.

Section 2 describes the partitioning procedure to identify separate wave systems from a $2 \mathrm{D}$ wave spectrum. Section 3 discusses the results of the azimuthal and radial variations of the wave propagation directions. Section 4 presents a parametric model of the dominant wave propagation direction and pdfs of multiwave directional properties. Section 5 is a summary.

\section{Wave system partitioning of $2 \mathrm{D}$ directional spectrum}

The archived SRA 2D wavenumber spectra $S\left(k_{E}, k_{N}\right)$ are stored as $65 \times 65$ matrices with element $(33,33)$ the spectral origin, where $k_{E}$ and $k_{N}$ are respectively the east and north components of the wavenumber vector; the 

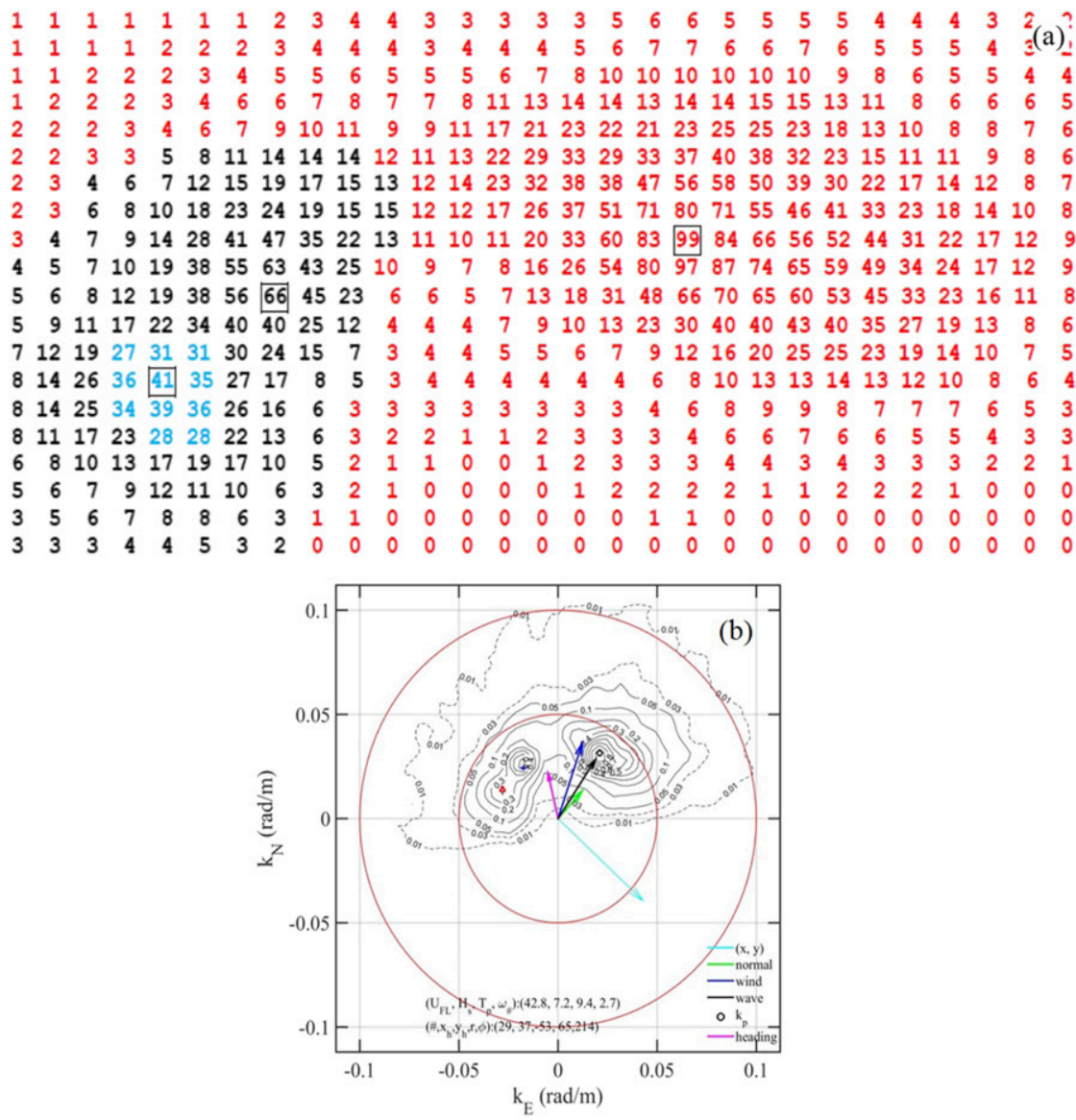

FIG. 1. An example of the $2 \mathrm{D}$ wavenumber spectrum used to illustrate the wave system partitioning. (a) A portion of the "printout" of the spectrum surrounding the area with high spectral values. The spectral values are normalized to integer numbers between 0 and 99 . Three wave systems are identified and shown with red, black, and cyan colors. (b) The contour map of the full 2D wavenumber spectrum, the location vector proportional to its distance from the hurricane center, the location vector normal, wind and dominant wave vectors, and hurricane heading are superimposed with arrows of different colors as labeled in the legend. The positions of the three local spectral peaks are shown in descending order with symbols circle, plus, and triangle. The Earth coordinates (E, N, W, and S) are used in the plotting. Additional information is given in the lower-left corner: line 1 is $\left(U_{\mathrm{FL}}, H_{s}, T_{p}, \omega_{\#}\right)$ and line 2 is spectrum sequence number, the position vectors $\left(x_{h}, y_{h}\right)$, and $(r, \phi)$ rotated with reference to the hurricane heading.

spectral resolution $d k$ is $0.0035 \mathrm{radm}^{-1}$. The spectral values are normalized and stored as integers ranging from 0 to 999. Figure 1a shows an example with the archived spectral values divided by 10 and rounded to nearest integers for this illustration. For clarity, only 29 columns and 20 rows surrounding the area with high spectral values are displayed. Figure $1 b$ shows the contour map of the complete normalized 2D spectrum using the full resolution of the archived spectral values. Multiple wave systems propagating in different directions can be easily detected.

To identify various wave systems, a partitioning algorithm was developed. The procedure first searches for local maxima, which are identified if, in a $3 \times 3$ subarea of the $2 \mathrm{D}$ wavenumber space, the maximum is located at the $(2,2)$ element of the subarea. Sliding the $3 \times 3$ mask through the whole $2 \mathrm{D}$ wavenumber space, the wavenumber locations of the local maxima are stored in an array. Additional criteria can be defined to remove small peaks by setting a threshold spectral value and to remove multiple local peaks in the same wave system by setting minimum distance between neighboring peaks. In the analysis presented in this paper, the threshold spectral peak level is set at $5 \%$ of the overall spectral maximum, and the minimum distance between neighboring peaks is $3 d k$. 
TABLE 1. Some basic information of the datasets used for the analysis in this paper. Umax, rm, Hsmax, Tpmax, \#S, \#T, Vh, and thN in the header represent the maximum flight level wind speed, radius of maximum wind, maximum significant wave height, maximum dominant wave period, number of spectra, number of radial transects, hurricane translation speed, and hurricane heading referenced to north (positive CCW) in each data file.

\begin{tabular}{|c|c|c|c|c|c|c|c|c|c|c|c|}
\hline File ID & $\begin{array}{l}\text { Umax } \\
\left(\mathrm{ms}^{-1}\right)\end{array}$ & $\begin{array}{l}\mathrm{rm} \\
(\mathrm{km})\end{array}$ & $\begin{array}{l}\text { Hsmax } \\
(\mathrm{m})\end{array}$ & $\begin{array}{l}\text { Tpmax } \\
\text { (s) }\end{array}$ & Start time & End time & ID & $\# S$ & $\# T$ & $\begin{array}{c}\mathrm{Vh} \\
\left(\mathrm{m} \mathrm{s}^{-1}\right)\end{array}$ & thN $\left(^{\circ}\right)$ \\
\hline Bonnie1998_24 & 45.7 & 74 & 10.9 & 13.3 & $\begin{array}{c}2029 \text { UTC } 24 \\
\text { Aug } 1998\end{array}$ & $\begin{array}{c}0144 \text { UTC } 25 \\
\text { Aug } 1998\end{array}$ & B24 & 233 & 10 & 4.5 & 13 \\
\hline Ivan2004_09 & 74.0 & 13 & 12.7 & 15.2 & $\begin{array}{c}1615 \text { UTC } 9 \\
\text { Sep } 2004\end{array}$ & $\begin{array}{c}2010 \text { UTC } 9 \\
\text { Sep } 2004\end{array}$ & I09 & 376 & 5 & 5.6 & 62 \\
\hline Ivan2004_12 & 59.5 & 17 & 12.0 & 13.8 & $\begin{array}{c}1039 \text { UTC } 12 \\
\text { Sep } 2004\end{array}$ & $\begin{array}{c}1541 \text { UTC } 12 \\
\text { Sep } 2004\end{array}$ & $\mathrm{I} 12$ & 456 & 6 & 4.3 & 65 \\
\hline Ivan2004_14 & 69.6 & 42 & 13.1 & 14.4 & $\begin{array}{l}2009 \text { UTC } 14 \\
\text { Sep } 2004\end{array}$ & $\begin{array}{c}0249 \text { UTC } 15 \\
\text { Sep } 2004\end{array}$ & I14 & 600 & 11 & 4.8 & 25 \\
\hline Bonnie1998_26 & 38.8 & 80 & 10.8 & 14.3 & $\begin{array}{c}1638 \text { UTC } 26 \\
\text { Aug } 1998\end{array}$ & $\begin{array}{c}2235 \text { UTC } 26 \\
\text { Aug } 1998\end{array}$ & B26 & 143 & 6 & 4.5 & 0 \\
\hline Floyd1999_13 & 61.4 & 32 & 12.2 & 14.2 & $\begin{array}{l}2012 \text { UTC } 13 \\
\text { Sep } 1999\end{array}$ & $\begin{array}{l}0051 \text { UTC } 14 \\
\text { Sep } 1999\end{array}$ & F13 & 194 & 5 & 5.0 & 70 \\
\hline Humberto2001_23 & 49.0 & 32 & 6.5 & 10.7 & $\begin{array}{l}2005 \text { UTC } 23 \\
\text { Sep } 2001\end{array}$ & $\begin{array}{c}2357 \text { UTC } 23 \\
\text { Sep } 2001\end{array}$ & $\mathrm{H} 23$ & 78 & 6 & 4.5 & -10 \\
\hline Humberto2001_24 & 36.9 & 45 & 6.0 & 10.8 & $\begin{array}{l}2054 \text { UTC } 24 \\
\text { Sep } 2001\end{array}$ & $\begin{array}{c}0058 \text { UTC } 25 \\
\text { Sep } 2001\end{array}$ & $\mathrm{H} 24$ & 86 & 6 & 6.5 & -50 \\
\hline Lili2002_30 & 36.7 & 75 & 5.7 & 11.2 & $\begin{array}{l}1922 \text { UTC } 30 \\
\text { Sep } 2002\end{array}$ & $\begin{array}{c}0003 \text { UTC } 1 \\
\text { Oct } 2002\end{array}$ & L20 & 163 & 4 & 4.0 & 45 \\
\hline Frances2004_31 & 70.2 & 32 & 12.1 & 14.7 & $\begin{array}{c}1641 \text { UTC } 31 \\
\text { Aug } 2004\end{array}$ & $\begin{array}{c}1922 \text { UTC } 31 \\
\text { Aug } 2004\end{array}$ & F31 & 113 & 3 & 5.8 & 80 \\
\hline Frances2004_01 & 62.5 & 32 & 10.0 & 14.5 & $\begin{array}{c}1639 \text { UTC } 1 \\
\text { Sep } 2004\end{array}$ & $\begin{array}{c}2011 \text { UTC } 1 \\
\text { Sep } 2004\end{array}$ & F01 & 212 & 5 & 5.5 & 70 \\
\hline
\end{tabular}

The next step is to identify the spectral components associated with each individual wave system defined by a local peak. In sequence starting from the highest peak, the eight peak-bordering spectral components are assigned to the wave system defined by the local peak. Extending outward from the local peak location, the neighboring spectral components with lower or equal values are assigned to the subject wave system. The process continues until all the spectral components not claimed by a previous wave system have been assigned.

Figure $1 \mathrm{~b}$ shows the result of local peaks identified by the spectral partitioning procedure described above. The local peaks (up to 5) are marked in descending order with circle, plus, triangle, square, and penta-star. For the example illustrated here there are three wave systems. Arrows with different colors as illustrated in the legend are added to show directions of the location vector, normal to the location vector, wind vector, dominant wave vector, and the hurricane heading. These color notations are used in all the directional spectral presentations in this paper.

In Fig. 1a, the spectral components belonging to the three wave systems are shown with different colors (red, black, and cyan), and the local peaks are outlined with rectangular boxes. Earth coordinates are used in Fig. 1.
In subsequent presentations of the directional spectrum, the coordinates will be rotated such that the hurricane heading is toward the top of the viewing perspective.

\section{Azimuthal and range variations}

Table 1 lists the 11 directional wavenumber spectrum datasets being used. The first four have been the subject of extensive investigations (Wright et al. 2001; Moon et al. 2003; Black et al. 2007; Fan et al. 2009b; Hwang 2016; Hwang and Walsh 2016; Hwang and Fan 2017; Hwang et al. 2017). The remaining seven include one collected during Bonnie 1998 landfalling (Walsh et al. 2002) and six previously unpublished [one each from Floyd (1999) and Lili (2002), and two each from Humberto (2001) and Frances (2004)].

Two of the datasets (B24 and I14) contain 10 and 11 radial transects that are more or less evenly distributed along the azimuth. Figure 2 shows the flight-level wind speed $U_{\mathrm{FL}}$ measured along the tracks and the interpolated 2D wind field; the method of $2 \mathrm{D}$ interpolation is further discussed in the appendix. The locations of maximum wind speed based on the aircraft data (marked with + ) are in the first and fourth quadrants for B24 and I14, respectively. The radial distance and 


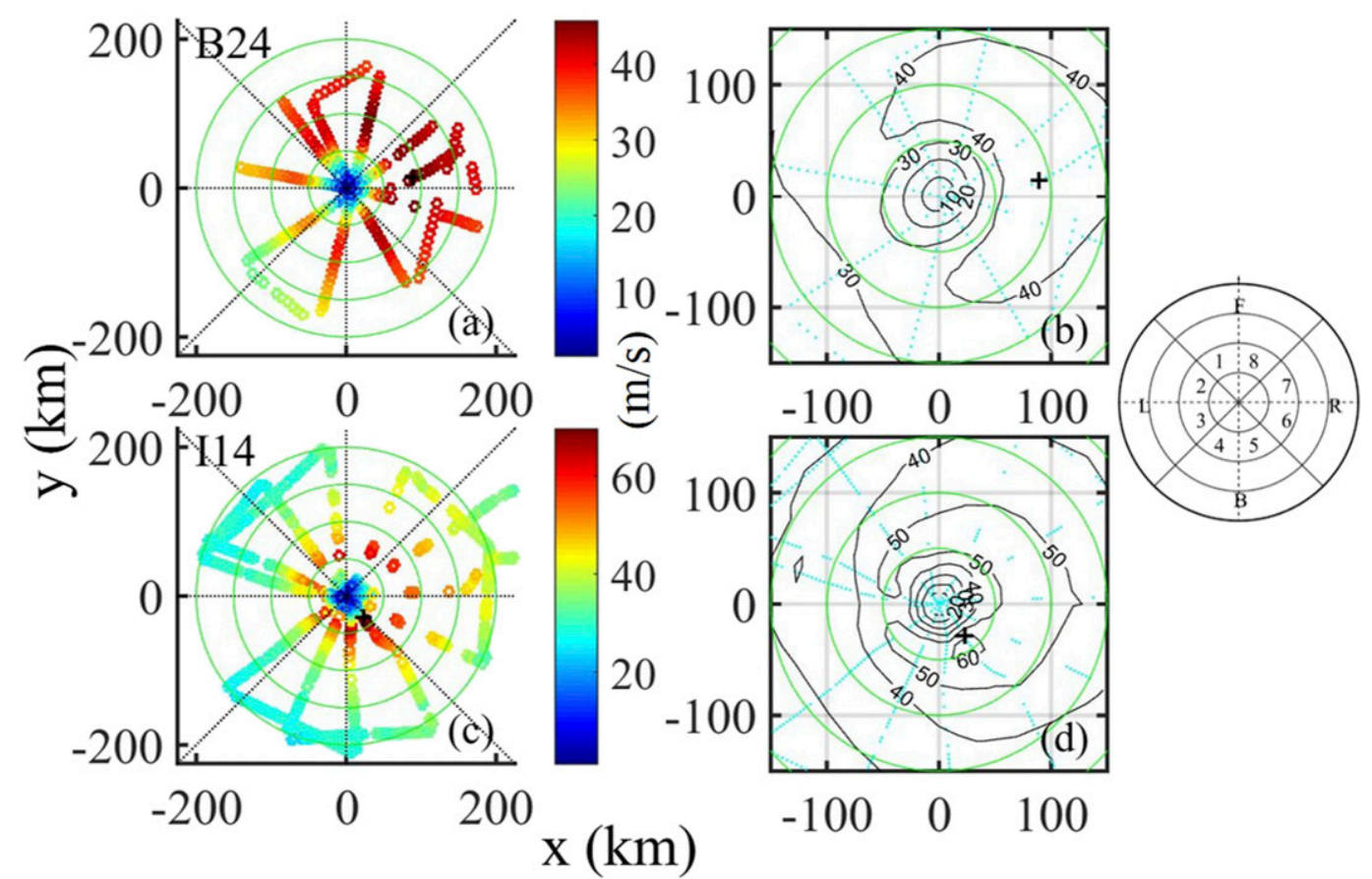

FIG. 2. The 2D wind fields of the (a),(b) B24 and (c),(d) I14 datasets used for the discussion of azimuthal and radial variations of wave propagation directions. Wind speed at the aircraft altitude is shown in the left panels and contours of the interpolated wind speed (zoomed in to show more details) are shown in the right panels. The superimposed circles are $50 \mathrm{~km}$ apart. The inset shows the eight azimuthal slices and four radial ranges for averaging the $2 \mathrm{D}$ spectra.

azimuth angle of maximum wind speed $\left(r_{m}, \phi_{m}\right)$ are $\left(90 \mathrm{~km}, 279^{\circ}\right)$ for B24, and $\left(36 \mathrm{~km}, 219^{\circ}\right)$ for I14. These $r_{m}$ values are different from those listed in Table 1. For the first four cases in Table 1, the more comprehensive NOAA 2D surface wind fields HWIND (Powell et al. 1996; Powell and Houston 1998; Dunion and Velden 2002; Dunion et al. 2002), closest to the aircraft data acquisition durations, were available; the listed $r_{m}$ from the 2D HWIND is considered to be more representative. More information on the aircraft wind data is given in the last paragraph of this section. Throughout this paper, all angles measured from a reference are positive counterclockwise $(\mathrm{CCW})$. The frequently used references in this paper are the hurricane heading, cardinal direction north, and location vector normal.

The two datasets provide a comprehensive spatial coverage of the wind and wave information inside TCs. The spectra are sorted into eight azimuthal slices $\left(0^{\circ}-\right.$ $45^{\circ}, 45^{\circ}-90^{\circ}, \ldots$ and $\left.315^{\circ}-360^{\circ}\right)$ and four equal radial divisions $(0-50,50-100,100-150$, and $150-200 \mathrm{~km})$ to give an average picture of azimuthal and radial variations of the wave propagation directions. The azimuth angle is measured from the hurricane heading and increases $\mathrm{CCW}$. The radial distance is measured from the hurricane center and increases outward. In subsequent discussions the azimuthal slices are numbered from 1 to 8 in the order as shown in the Fig. 2 inset.

The results of the directional spectrum spatial patterns are displayed in Figs. 3 and 4 for B24 and I14, respectively. In each figure, panels (a)-(d) are for the four equal radial divisions: $0-50,50-100,100-150$, and $150-200 \mathrm{~km}$, respectively. Because the region around the radius of maximum wind (RMW) is of great interest, we also show the result for the radial division between $0.5 r_{m}$ and $1.5 r_{m}$ in panel (e).

For each radial division, azimuthal slices 1-4 are arranged on the left-hand side from top to bottom, and slices 5-8 are on the right-hand side from bottom to top. These positions correspond to the left/right (left four/ right four) and front/back (top four/bottom four) of the eight slices referenced to the hurricane heading. The number of spectra averaged for each radial-azimuthal sector is shown in parentheses next to the azimuthal slice number at the upper-left corner. The average flight-level wind speed, significant wave height, and dominant wave period are shown at the top of each spectrum. The illustrated directional spectrum is normalized so the maximum value is unity. For clarity, there are only five contour lines relative to the spectral peak $(0.1,0.3, \ldots$, $0.9)$. The two circles correspond to $k=0.025$ and 


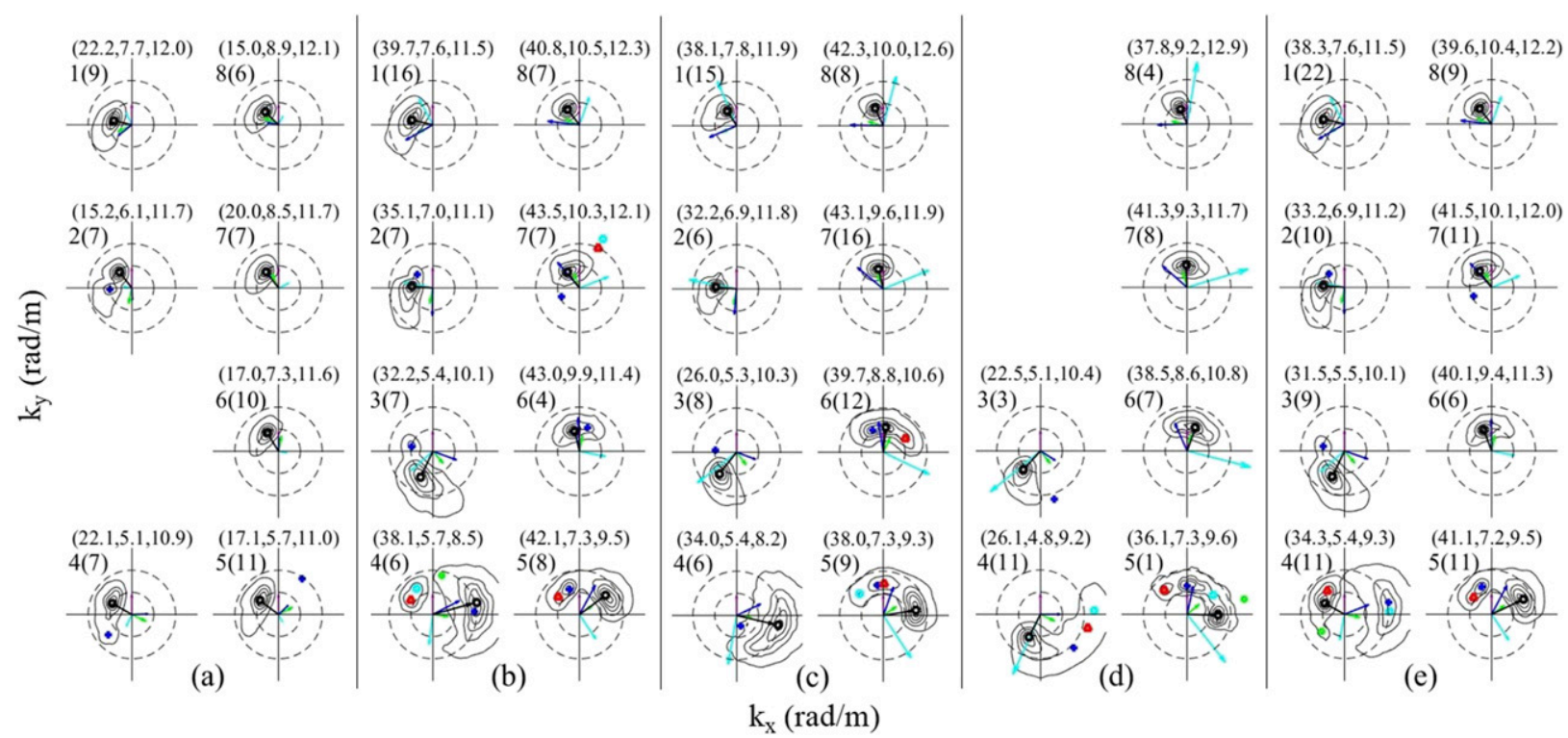

FIG. 3. The average directional spectra of the B24 dataset in four equal radial divisions: (a) 0-50 km, (b) 50-100 km, (c) 100-150 km, and (d) $150-200 \mathrm{~km}$. In addition, (e) shows the result for the radial division between $0.5 r_{m}$ and $1.5 r_{m}$. Each radial division is subdivided into eight azimuthal slices; the slice number is shown at the upper-left corner, and the number of spectra in the average is given in parentheses next to the slice number. The two circles are at $k=0.025$ and $0.05 \mathrm{rad} \mathrm{m}^{-1}$. Blank space indicates no data in the given azimuth-range sector. Further details are given in the text.

$0.05 \mathrm{rad} \mathrm{m}^{-1}$. The arrows show the location vector, location vector normal, wind vector, dominant wave vector, and hurricane heading, with identical color coding as that of Fig. 1, and the coordinates are rotated so the heading is toward the top of the viewing perspective.

Several features of the wave propagation directions are revealed from the analysis:
1) Directionally monomodal spectra are dominant in slices $1,2,3,7$, and 8 of the hurricane coverage area (the front half and the front part of the left-back quarter).

2) Directionally multimodal spectra are dominant in slices 4-6 (the right-back quarter and the rear part of the left-back quarter) outside the RMW.

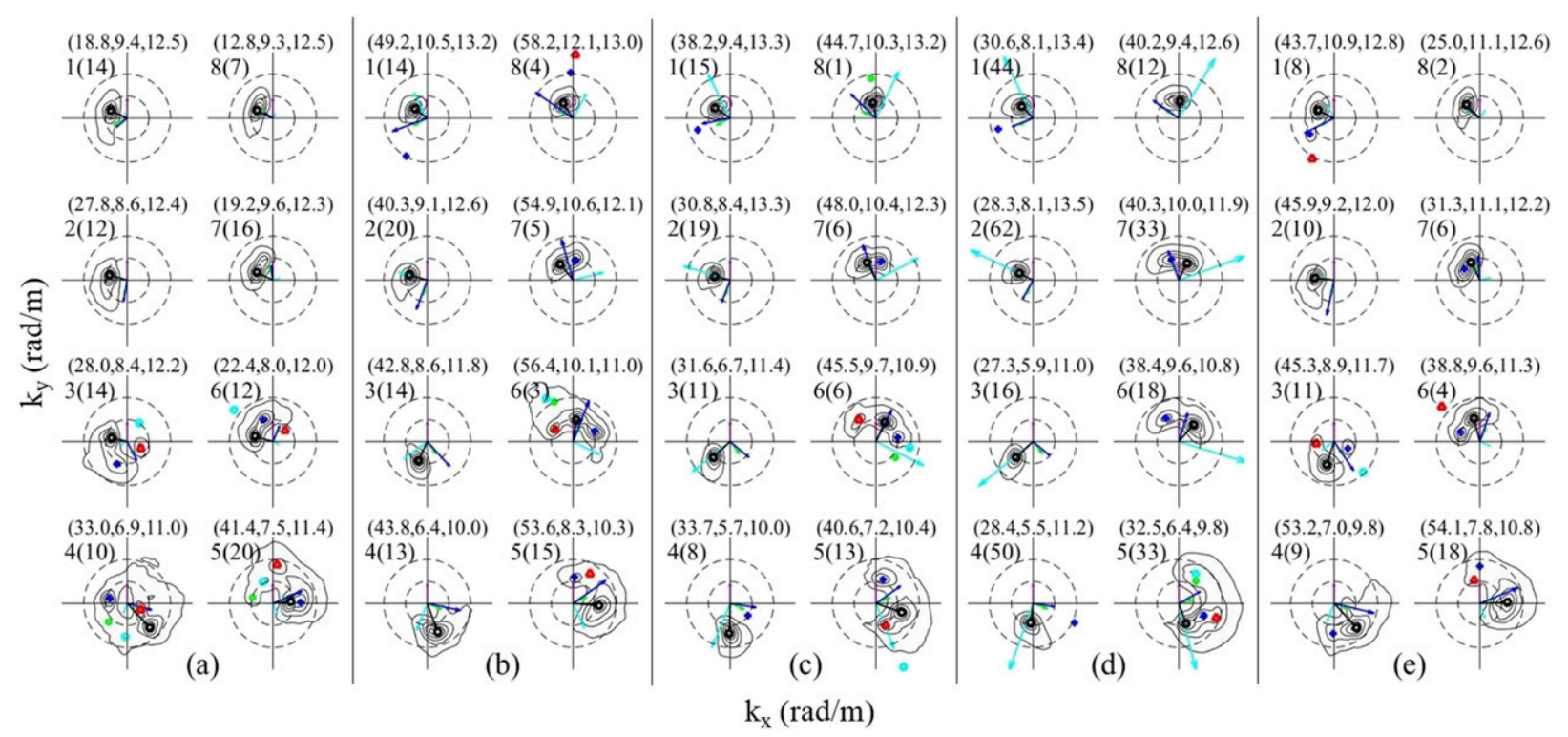

FIG. 4. As in Fig. 3, but for the I14 dataset. 

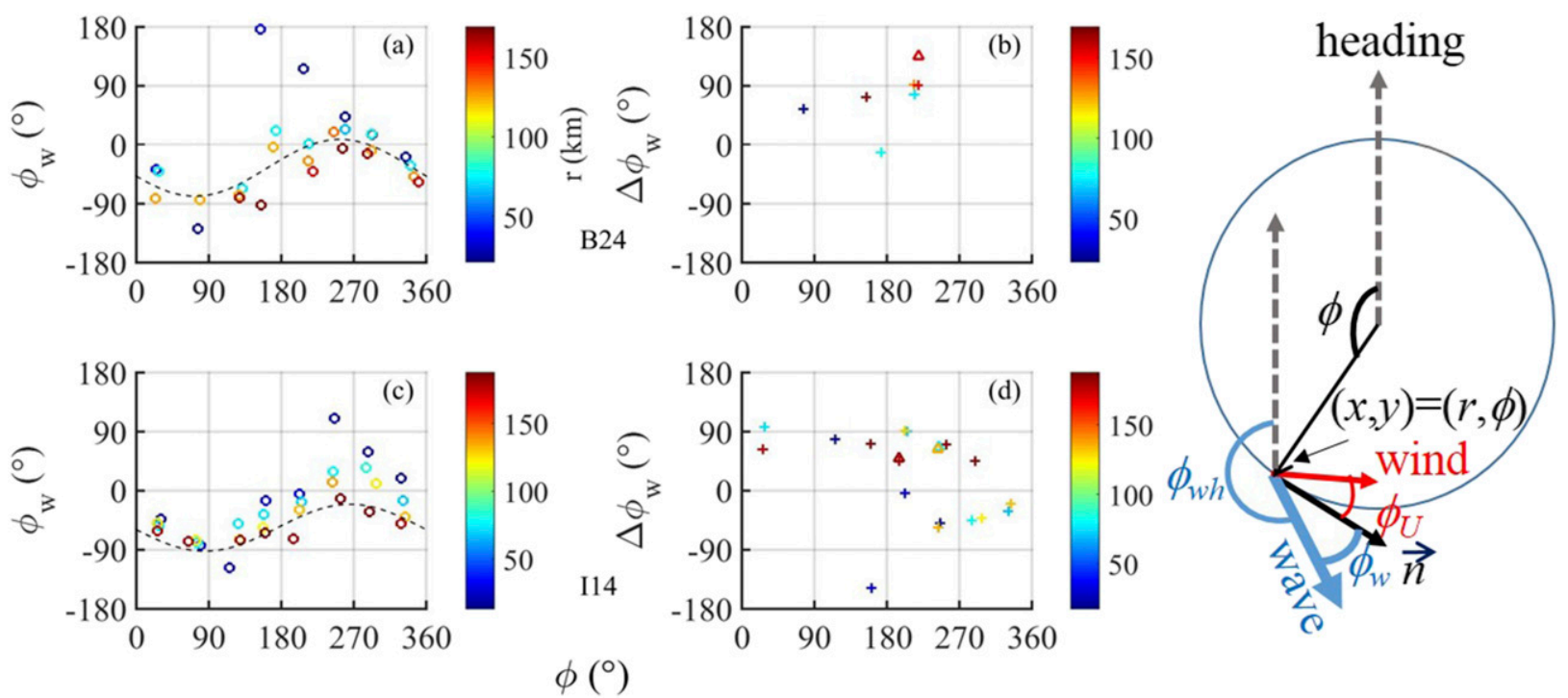

FIG. 5. Summary results of range and azimuthal variations: (a),(c) the dominant wave propagation direction and (b),(d) directional differences of the secondary (plus marker) and tertiary (triangle marker) wave systems from the primary wave system. The color coding is radial distance. The smooth dashed lines in the left column are best-fit curves through the data within $50 \leq r \leq 200 \mathrm{~km}$ given in Hwang et al. (2017). The inset describes the directional variables.

3) The primary wave propagation direction in the region near the hurricane center is toward front left relative to the hurricane heading.

4) Away from the hurricane center, variations of the wave propagation directions are primarily azimuth dependent; the radial dependency is relatively weak. Further detail of the radial and azimuthal dependency is given in Fig. 5 and section 4.

5) Backward propagating waves are in slices 3-5 in regions including or outside the RMW.

6) Overall, there is a leftward and frontward trend of wave propagation inside the hurricane coverage area, except in slices 3 and 4 outside the RMW.

7) Although there are some differences, the wave directional pattern in the ring around the RMW behaves very similarly to those outside the RMW.

8) There appears to be some effect of $\phi_{m}$ (the azimuth angle of the maximum wind speed location) on the overall directional pattern of wave propagation. Given the poor azimuthal resolution in most of the datasets (with six or fewer radial transects; Table 1), the effect of $\phi_{m}$ on wave propagation direction is not resolved at this time.

The directional results presented in Figs. 3 and 4 are summarized in Fig. 5, showing the dominant wave direction in the left column and the relative angles between the secondary and tertiary wave systems from the primary system in the right column. We only account for those secondary and tertiary wave systems with at least $10 \%$ of the variance of the primary wave system. The wave angle is measured from the location vector normal as indicated in the inset of Fig. 5. The results for B24 are shown in the top row and those for I14 are shown in the bottom row. Except for the region close to the hurricane center, the primary feature of the dominant wave propagation direction is the sinusoidal azimuthal variation; the radial dependency is characterized by a nearly constant offset of the sinusoidal curves (Figs. 5a,c). The smooth dashed lines in the left column are best-fit curves through the data within $50 \leq r \leq 200 \mathrm{~km}$ given in Hwang et al. (2017). For the observations very close to the hurricane center, the waves propagate mainly toward the front-left direction relative to the hurricane heading (Figs. 2a, 3a), and thus the $\phi_{w}$ dependency on $\phi$ is almost linear (the deep blue symbol in Figs. 5a and 5c; more detail is shown in Fig. 6 in section 4). The wave direction measured from the hurricane heading $\phi_{w h}$ (in degrees) is related to $\phi_{w}$ by $\phi_{w h}=\phi_{w}+(\phi+90)$, where $\phi+90$ is the angle of the location vector normal (see inset of Fig. 5).

The directionally multimodal spectrum occurs mainly in the back half plane, that is, $\phi$ between $90^{\circ}$ and $270^{\circ}$ (Figs. 5b,d). The plotted directional difference is defined as $\Delta \phi_{w j i}=\Delta \phi_{w j}-\Delta \phi_{w i}$, where the subscript $i$ or $j$ is the sequence number of the multiple spectral peaks. The plus marker shows $\Delta \phi_{w 21}$ and the triangle marker is for $\Delta \phi_{w 31}$. Most minor wave systems propagate in the lefthand side $(\mathrm{CCW})$ of the primary system (positive $\Delta \phi_{w 21}$ and $\Delta \phi_{w 31}$ ), with large angle difference (mostly between about $60^{\circ}$ and $90^{\circ}$ ). In section 4 , we present more details 


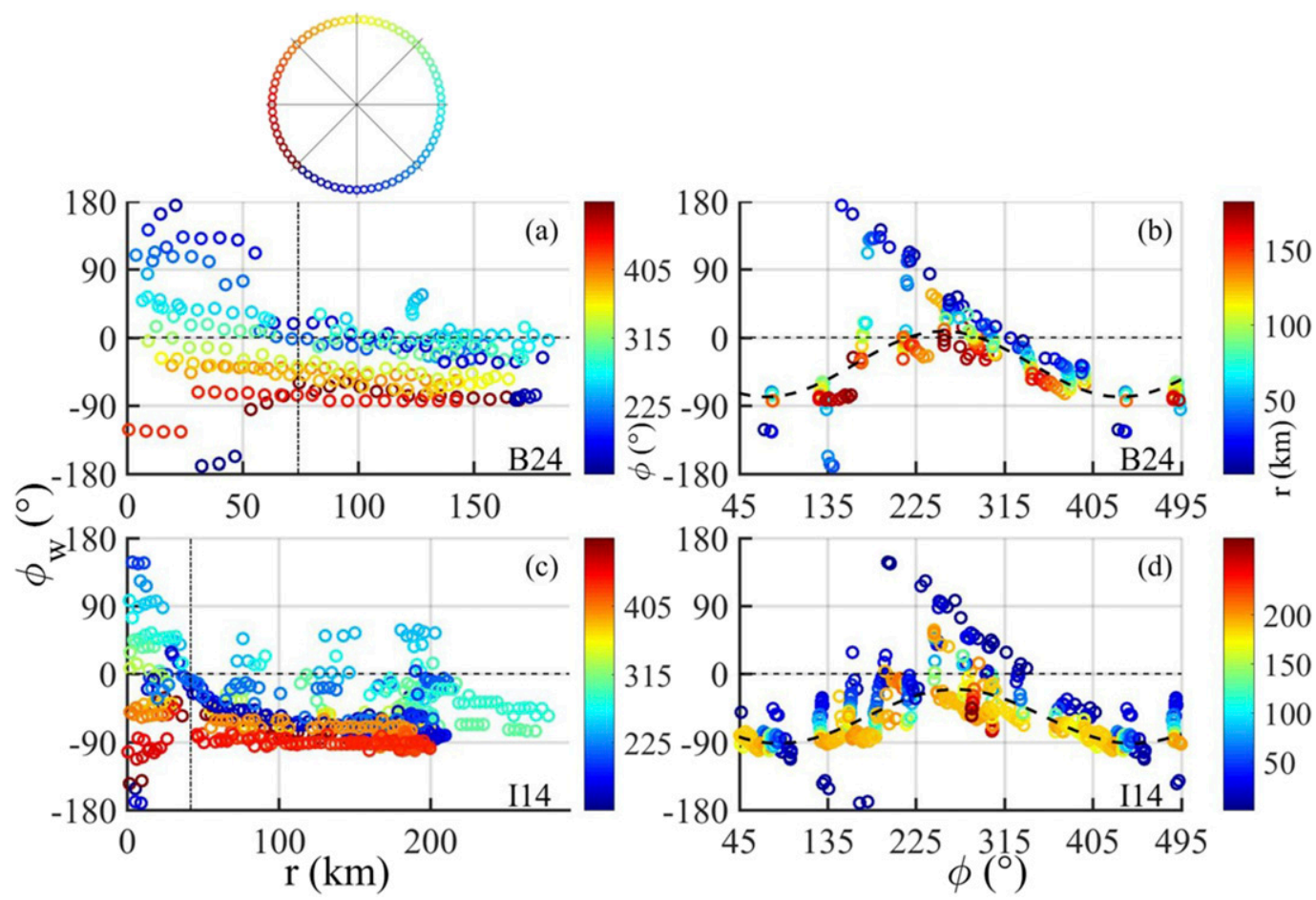

FIG. 6. Dependency of dominant wave direction on (a),(c) radial distance and (b),(d) azimuth angle. The top row shows the results of B24 dataset, and the bottom row shows those of I14 dataset. The color coding is azimuthal angle for the left column (including the inset) and radial distance for the right column. The smooth dashed lines in the right column are best-fit curves through the data within $50 \leq r \leq 200 \mathrm{~km}$ given in Hwang et al. (2017).

on the spatial variation and multisystem properties obtained from the directional analysis without rangeazimuth averaging.

There are more cases of multimodal spectra observed in I14 than B24. At the time of measurement, I14 is at category 4 with the maximum wind location in the rightback quarter, and B24 is at category 2 with the maximum wind location in the right-front quarter. These differences in the hurricane characteristics may have contributed to the observed differences in the properties of the multiwave spectra. As mentioned earlier, the azimuthal resolution is very coarse in most of the datasets (with six or fewer radial transects; Table 1). The correct determination of maximum wind location, especially the azimuth angle, is difficult from the available data. We are unable to clarify the effects of $\phi_{m}$ on the wave propagation direction at this stage.

The datasets listed in Table 1 report the flight-level east and north wind components, which were interpreted incorrectly as the surface wind in three earlier analyses (Hwang and Walsh 2016; Hwang and Fan 2017; Hwang et al. 2017). The relationship between flight level and surface $(10 \mathrm{~m})$ wind velocities is of great interest, and significant improvement in the vertical wind profile has been achieved with the use of the global positioning system (GPS) dropwindsondes initiated in 1997 (e.g., Powell 1980, 1982; Dunion et al. 2003; Franklin et al. 2003; Uhlhorn and Black 2003; Uhlhorn et al. 2007). For example, Table 2 of Franklin et al. (2003) lists the recommended operational wind adjustment factor $R_{U}=$ $U_{10} / U_{\mathrm{FL}}$ for reconnaissance flight-level winds to the surface in the eyewall and the outer vortex. For 700- and 850-hPa flight levels (about 3100 and $1500 \mathrm{~m}$, respectively), within which most wave measurements were performed, $R_{U}=0.90$ and 0.80 for the eyewall and 0.85 and 0.80 for the outer vortex. The correction factor for the flight level wind directions to the surface is less certain. Examples of the measured flight level wind directions are given in the appendix. The effects on the determination of the effective fetch or duration of the TC wind fields derived by using the flight level versus surface wind speeds are quantified in section 5 of Hwang and Walsh (2018).

\section{Wave direction model}

\section{a. Dominant wave system}

The directional wave spectra provide detailed information on the azimuthal and radial variations of 

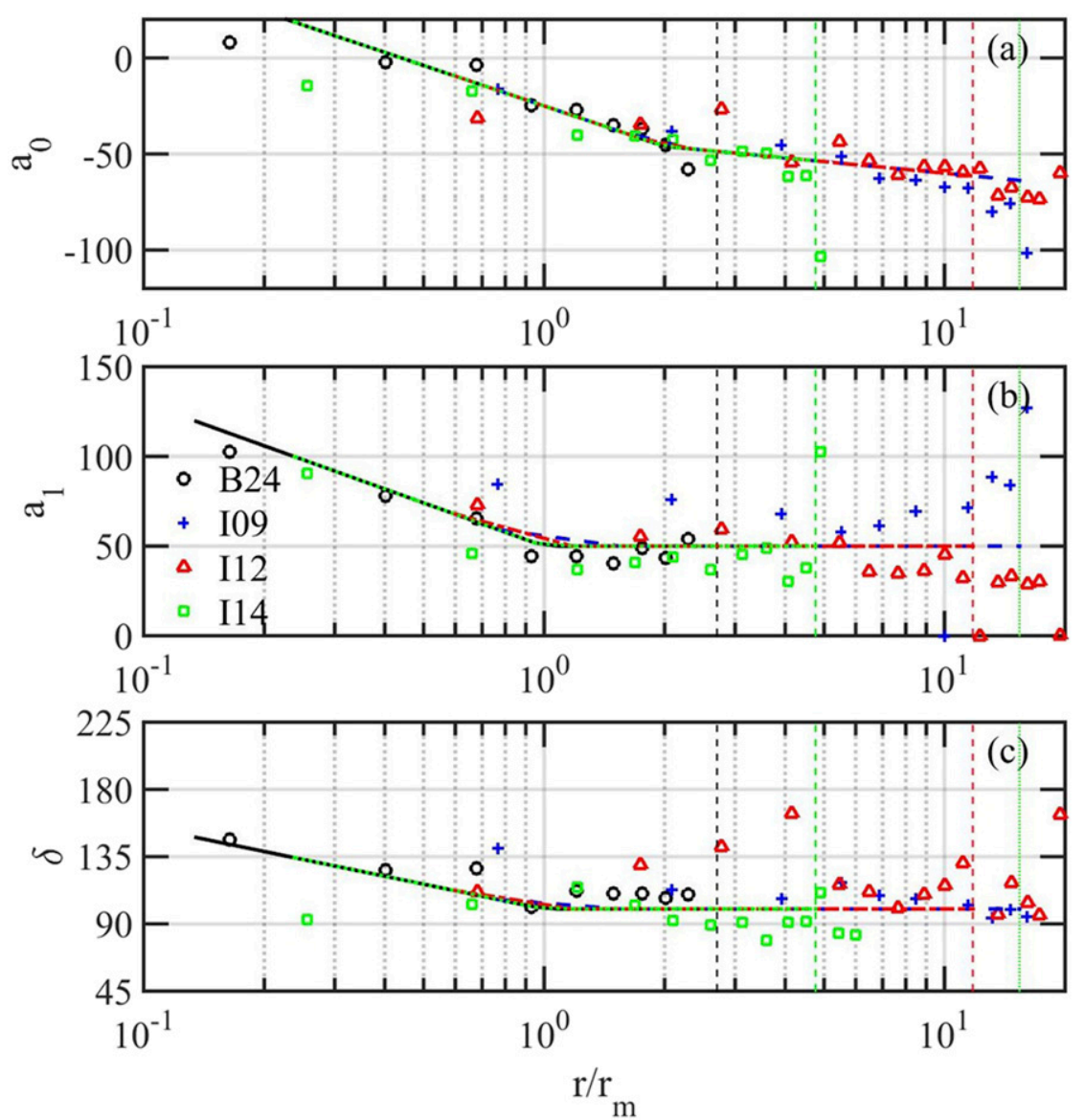

FIG. 7. Radial dependency of the best-fit coefficients of the sinusoidal function (1): (a) $a_{0}$, (b) $a_{1}$, and (c) $\delta$. Results based on the first four datasets in Table 1 are shown.

surface wave propagation inside tropical cyclones. Here we present a model of dominant wave direction based on the measured wave data without azimuthal and radial averaging as performed in section 3 . The left and right columns of Fig. 6 show the dependency of the dominant wave angle $\phi_{w}$ on the radial distance $r$ and the azimuth angle $\phi$ of the measurement location, respectively. The results for B24 are given in the top row and those for I14 are in the bottom row. For this figure, the azimuth angle color coding (left column) is arranged such that the number on the color map increases in the order of back, right, front, and left quarters as illustrated in the inset, that is, the $\phi$ range for the display is from $135^{\circ}$ to $495^{\circ}$ as shown in the color bars in the left column. This sequence corresponds approximately to the order of increasing wave age (Hwang 2016; Hwang and Walsh 2016; Hwang and Fan 2017).

The radial dependence (left column) is relatively weak outside $r_{m}$, which is shown with a vertical dashed line in the figure. Since $\phi_{w}$ is measured from the location vector normal, $\phi_{w}=0^{\circ}$ means that the waves are propagating in the local tangential direction $(\mathrm{CCW})$ with respect to the circle defined by the local position and the hurricane center, and $\phi_{w}=-90^{\circ}\left(90^{\circ}\right)$ means waves are propagating radially outward (inward). (All angles are positive $\mathrm{CCW}$ from reference.)

Outside the RMW, consistent features between B24 and I14 include the following: (i) in the right quarter (light blue circles), the waves propagate along the local tangential; (ii) in the left quarter (red circles), the waves propagate radially outward; and (iii) in the front quarter (yellowish circles), the waves propagate toward the right-hand side of the position vector (outflow) at a large angle $\left(\phi_{w}\right.$ between about $-45^{\circ}$ and $\left.-70^{\circ}\right)$ with an increasing trend of the angle magnitude away from the hurricane center. The major directional differences between B24 and I14 occur in the back quarter (deep blue circles). For B24, the wave direction in the back quarter behaves similar to that in the right quarter, whereas for I14, it is similar to the left or front quarter. Another difference between the two cases is that outside the RMW, there are many more inflow cases observed in I14 


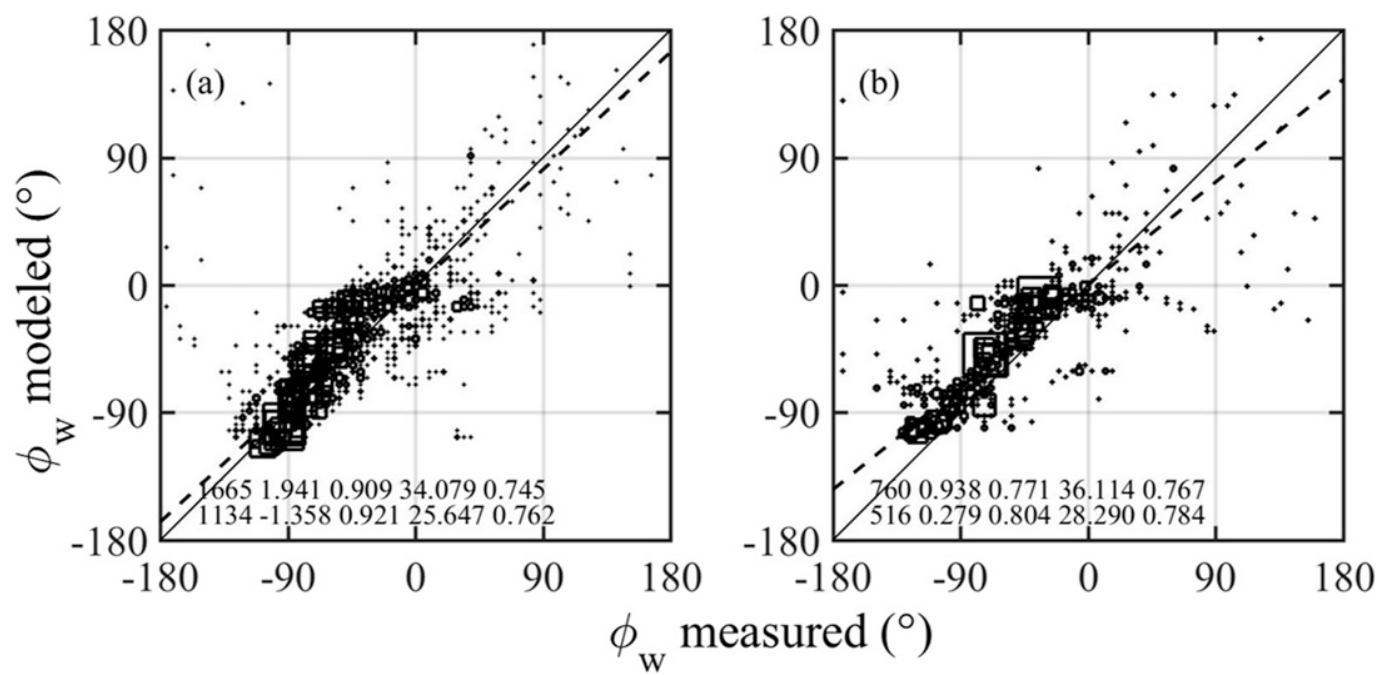

FIG. 8. Comparison of the modeled and measured dominant wave directions: results from (a) data used for model development and (b) data not used for model development (excluding B26 and H24 datasets). The statistics of the number of data, bias, slope of linear regression, rms difference, and correlation coefficient $\left(N, b_{0}, b_{1}, b_{2}\right.$, and $\left.b_{3}\right)$ for the whole population and subpopulation within $50 \leq r \leq 200 \mathrm{~km}$ are shown in the upper and lower text strings. The best-fit curves of the subpopulation are shown with dashed curves. The solid lines indicate perfect agreement. The size of the plotting symbol is proportional to the data density at the plotting coordinates.

than in $\mathrm{B} 24\left(\phi_{w}>0^{\circ}\right.$; the waves propagate toward the left-hand side of the position vector normal, thus toward the hurricane center). As mentioned in section 3, I14 is category 4 with maximum wind located in the right-back quarter, and B24 is category 2 with maximum wind located in the right-front quarter. It is plausible that the azimuth angle of the maximum wind location plays a prominent role in the observed directional difference in the back quarter, but the impact of hurricane intensity cannot be ruled out.

The azimuthal dependence (right column) is close to sinusoidal for data with $r>r_{m}$. Very close to the hurricane center, the dominant waves propagate mainly toward the front-left direction relative to the hurricane heading, so $\phi_{w h}$ is almost constant (Figs. 3a, 4a), and thus the $\phi_{w}$ dependency on $\phi$ is almost linear: $\phi_{w h}=$ $\phi_{w}+(\phi+90)$, as shown by the data with deep blue circles in Figs. $6 \mathrm{~b}$ and $6 \mathrm{~d}$. The linear trend is also detectable in Figs. 5a and 5c, which are based on eight azimuthal slices and four radial divisions and are thus of much coarser resolution.

The superimposed dashed curves in Figs. $6 \mathrm{~b}$ and $6 \mathrm{~d}$ are the best-fit sinusoidal functions derived from data within $50 \leq r \leq 200 \mathrm{~km}$ (Hwang et al. 2017):

$$
\phi_{w}=a_{0}+a_{1} \cos (\phi+\delta) .
$$

Refining the radial resolution to $20 \mathrm{~km}$ for least squares fitting, the fitted coefficients $a_{0}, a_{1}$, and $\delta$ (in degrees) are shown as functions of the normalized radial distance $r_{*}=r / r_{m}$ in Fig. 7 for the first four datasets listed in Table 1. Although data scatter is large, there appears to be a consistent trend of radial dependency in the fitted coefficients. The superimposed smooth curves are

$$
\begin{aligned}
a_{0} & =\left\{\begin{array}{ll}
-25-70 \log r_{*}, & r_{*} \leq 2 \\
-40-20 \log r_{*}, & r_{*}>2
\end{array},\right. \\
a_{1} & =\left\{\begin{array}{ll}
50-80 \log r_{*}, & r_{*} \leq 1 \\
50, & r_{*}>1
\end{array},\right. \\
\delta & =\left\{\begin{array}{ll}
100-55 \log r_{*}, & r_{*} \leq 1 \\
100, & r_{*}>1
\end{array} .\right.
\end{aligned}
$$

Table 2 summarizes the results of comparing the modeled and measured wave directions for the 11 datasets. The listed statistics include the number of data points $N$, bias $b_{0}$, slope of linear regression $b_{1}$, root-meansquare (rms) difference $b_{2}$, and correlation coefficient $b_{3}$. Also tabulated are the hurricane translation speed $V_{h}$ and direction $\phi_{h}$ (relative to north, increasing $\mathrm{CCW}$ ) derived from best-track information (NOAA HURDAT2; http:// www.aoml.noaa.gov/hrd/hurdat/Data_Storm.html). The listed $r_{m}$ is estimated from the aircraft data except for the first four cases, for which the $r_{m}$ is from HWIND as discussed earlier in section 3. For each dataset, the statistics computed for the whole population are shown in the first line, and those from the subpopulation within $50 \leq r \leq 200 \mathrm{~km}$ are shown in the second line. 
TABLE 2. Regression statistics comparing the modeled and measured dominant wave propagation directions and best-track information. For each dataset, the first line includes all data points and the second line is for data within $50 \leq r \leq 200 \mathrm{~km}$. The listed statistics include $N, b_{0}, b_{1}, b_{2}, b_{3}, V_{h}, \phi_{h}$, and $r_{m}$, which are the number of data points, bias, slope of linear regression, rms difference, correlation coefficient, hurricane translation speed, hurricane heading referenced to north (positive $\mathrm{CCW}$ ), and radius of maximum wind speed, respectively. Combined data group G1 includes B24, I09, I12 and I14, and group G2 includes F13, H23, L30, F31, and F01 (Table 1).

\begin{tabular}{|c|c|c|c|c|c|c|c|c|}
\hline ID & $N$ & $b_{0}\left(^{\circ}\right)$ & $b_{1}$ & $b_{2}\left(^{\circ}\right)$ & $b_{3}$ & $V_{h}\left(\mathrm{~m} \mathrm{~s}^{-1}\right)$ & $\phi_{h}\left({ }^{\circ} \mathrm{N}\right)$ & $r_{m}(\mathrm{~km})$ \\
\hline B24_1 & 233 & 2.86 & 0.86 & 41.66 & 0.74 & 4.5 & 13 & 74 \\
\hline B24_2 & 176 & -2.33 & 0.89 & 24.30 & 0.80 & 4.5 & 13 & 74 \\
\hline I09_1 & 376 & -7.13 & 0.79 & 32.99 & 0.77 & 4.8 & 62 & 13 \\
\hline I09_2 & 311 & -7.35 & 0.80 & 31.04 & 0.75 & 4.8 & 62 & 13 \\
\hline I12_1 & 456 & 3.00 & 1.01 & 24.54 & 0.82 & 4.3 & 65 & 17 \\
\hline I12_2 & 212 & 0.32 & 1.05 & 23.89 & 0.76 & 4.3 & 65 & 17 \\
\hline I14_1 & 600 & 7.12 & 0.91 & 37.54 & 0.70 & 5.6 & 25 & 42 \\
\hline I14_2 & 435 & 2.78 & 0.95 & 22.56 & 0.78 & 5.6 & 25 & 42 \\
\hline B26_1 & 143 & -13.45 & 0.41 & 58.63 & 0.54 & 4.5 & 0 & 74 \\
\hline B26_2 & 71 & -15.27 & 0.38 & 56.03 & 0.45 & 4.5 & 0 & 74 \\
\hline F13_1 & 194 & -4.91 & 0.90 & 29.62 & 0.80 & 5.0 & 70 & 32 \\
\hline F13_2 & 118 & 0.82 & 1.00 & 15.82 & 0.87 & 5.0 & 70 & 32 \\
\hline H23_1 & 78 & 3.27 & 0.69 & 40.00 & 0.82 & 4.5 & -10 & 32 \\
\hline $\mathrm{H} 23 \_2$ & 59 & 4.27 & 0.72 & 33.65 & 0.84 & 4.5 & -10 & 32 \\
\hline H24_1 & 86 & -21.82 & 0.40 & 92.83 & 0.46 & 6.5 & -50 & 45 \\
\hline $\mathrm{H} 24 \_2$ & 58 & -28.06 & 0.35 & 100.25 & 0.28 & 6.5 & -50 & 45 \\
\hline L30_1 & 163 & 0.44 & 0.79 & 42.33 & 0.73 & 4.0 & 45 & 75 \\
\hline L30_2 & 106 & -5.38 & 0.76 & 35.49 & 0.82 & 4.0 & 45 & 75 \\
\hline F31_1 & 113 & 8.10 & 0.76 & 28.12 & 0.94 & 5.8 & 80 & 32 \\
\hline F31_2 & 62 & 3.77 & 0.78 & 21.18 & 0.98 & 5.8 & 80 & 32 \\
\hline F01_1 & 212 & 1.39 & 0.71 & 38.53 & 0.65 & 5.5 & 70 & 25 \\
\hline F01_2 & 171 & 1.85 & 0.78 & 30.13 & 0.53 & 5.5 & 70 & 25 \\
\hline \multicolumn{9}{|c|}{ Data used for model } \\
\hline G1_1 & 1665 & 1.94 & 0.91 & 34.08 & 0.75 & & & \\
\hline G1_2 & 1134 & -1.36 & 0.92 & 25.65 & 0.76 & & & \\
\hline \multicolumn{9}{|c|}{ Data not used for model, excluding B26 and $\mathrm{H} 24$} \\
\hline G2_1 & 760 & 0.94 & 0.77 & 36.11 & 0.77 & & & \\
\hline G2_2 & 516 & 0.28 & 0.80 & 28.29 & 0.78 & & & \\
\hline
\end{tabular}

Overall, the comparison statistics are relatively consistent. Except for two datasets (B26 and H24), the bias is less than $9^{\circ}$, the linear regression slope is between 0.7 and 1.1 , the rms difference is between $16^{\circ}$ and $42^{\circ}$, and the correlation coefficient is better than 0.7 in eight out of the nine remaining datasets.

A couple of reasons causing the large deviation between measured and modeled wave propagation directions are suggested here for the two outlier datasets:

(a) B26 is Bonnie (1998) at landfalling, and the land proximity appears to be the reason for the large differences between the observed wave direction and the calculation by model, which is based on four deepwater datasets (B24, I09, I12, and I14). The proximity of land would have altered the structure of the wind field and hence the waves; the associated bottom bathymetry could also influence wave propagation.

(b) H23 and H24 are Humberto (2001) following path recurvature. Based on the HURDAT2 analysis, the recurvature started at about 1200 UTC
23 September 2001 . The data collection time is 20052357 UTC 23 September for H23 and 2054 UTC 24 September to 0058 UTC 25 September for H24. The hurricane translation direction increased rapidly from $-10^{\circ}$ to $-50^{\circ} \mathrm{N}$ in about one day, and the translation speed also increased from 4.5 to $6.5 \mathrm{~m} \mathrm{~s}^{-1}$ during the same period. (All angles are positive CCW from reference.) Interestingly, the statistics of $\mathrm{H} 23$ are similar to the majority datasets but those of H24 are clearly much worse (Table 2).

Figure 8 shows the comparison of the modeled and measured dominant wave propagation directions. The results combining the four datasets used for the model development are given in Fig. 8a, and those from combining five of the seven datasets not used for the model development (excluding B26 and H24) are given in Fig. $8 \mathrm{~b}$. The dashed lines are the best-fit linear curves and solid lines indicate perfect agreement. The modelmeasurement comparison statistics of the combined datasets are also given in Table 2 as G1 and G2 and 


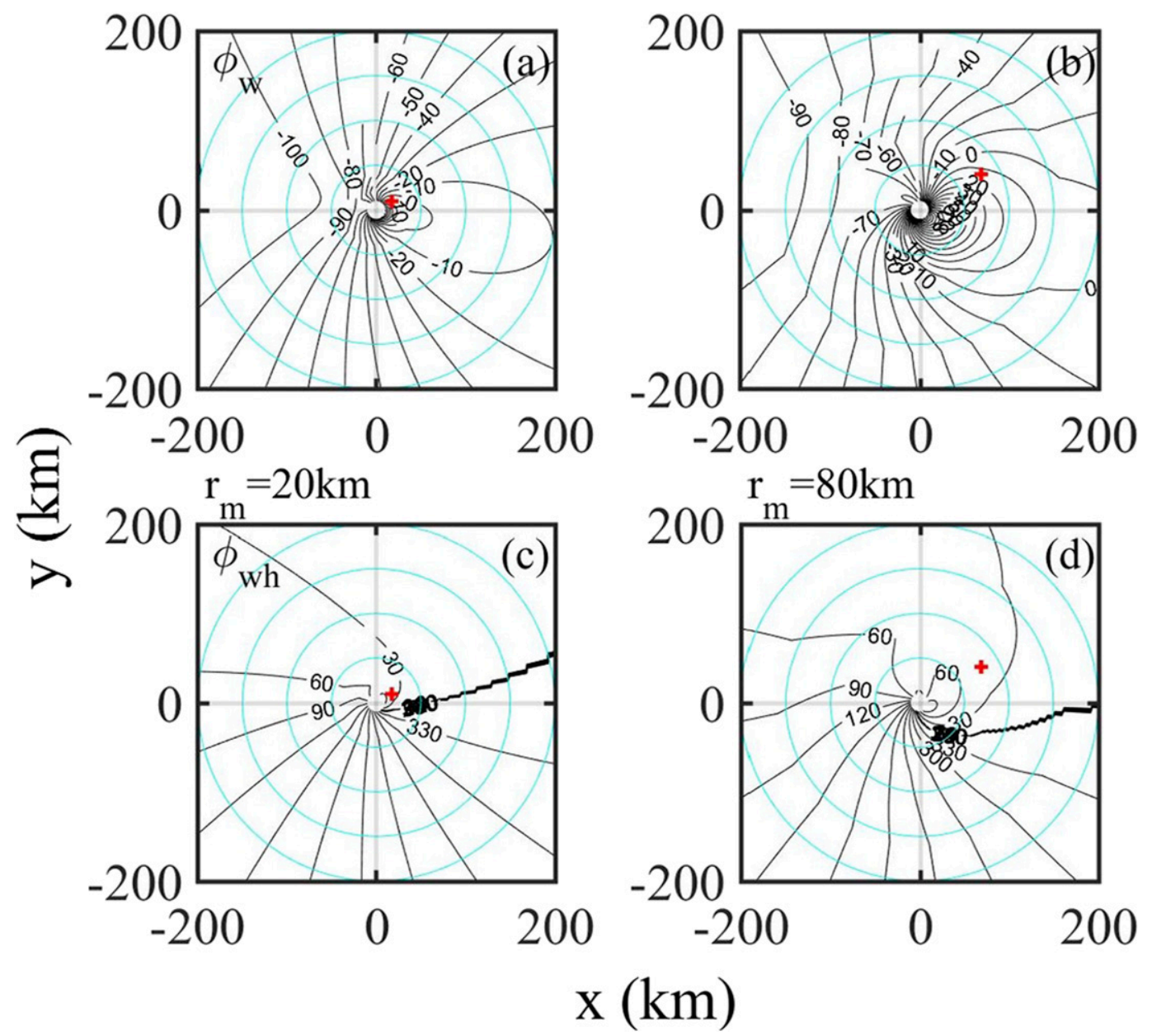

FIG. 9. Examples of the modeled wave propagation directions inside hurricanes: (a),(c) $r_{m}=20 \mathrm{~km}$ and (b),(d) $r_{m}=$ $80 \mathrm{~km}$. The top row shows $\phi_{w}$, the wave direction measured from the location vector normal, and the bottom row shows $\phi_{w h}$, the wave direction measured from the hurricane heading. The contours are $10^{\circ}$ apart for $\phi_{w}$ and $30^{\circ}$ apart for $\phi_{w h}$. Light-colored circles are $50 \mathrm{~km}$ apart. The location of maximum wind speed is shown with a plus marker.

printed near the bottom edge of the two figures. For data within $50 \leq r \leq 200 \mathrm{~km}$, the bias is less than $1.4^{\circ}$; for $\mathrm{G} 1$ and $\mathrm{G} 2$ the slopes of linear regression are 0.92 and 0.80 , respectively; the rms differences are $26^{\circ}$ and $28^{\circ}$; and the correlation coefficients are 0.76 and 0.78 . The statistics accounting for the whole group populations are only slightly worse.

Figure 9 shows examples of the modeled wave propagation directions inside hurricanes with $r_{m}=20$ and $80 \mathrm{~km}$. The top row shows $\phi_{w}$ the wave direction measured from the location vector normal; the bottom row shows $\phi_{w h}$ the wave direction measured from the hurricane heading. The results for $r_{m}=20 \mathrm{~km}$ are shown in the left column, and those for $r_{m}=80 \mathrm{~km}$ are in the right column. The contours are $10^{\circ}$ apart for $\phi_{w}$ and $30^{\circ}$ apart for $\phi_{w h}$. Two regions of special interest are discussed below.
The first region of special interest is in the right and front with $\phi_{w h}$ between about $0^{\circ}$ and $60^{\circ}$. In this region the generated waves propagate into the advancing wind field, and thus they can be expected to receive extended wind forcing leading to enhanced growth. For hurricanes with large $r_{m}$ (Fig. 9d), this region is in the first quadrant (front-right quarter) and extending somewhat on both ends of the first quadrant; for hurricanes with small $r_{m}$ (Fig. 9c), the region encompasses almost the entire front half plane. In essence, for hurricanes with smaller $r_{m}$, the region of waves propagating forward into the advancing wind field is broader, so the effective fetch or duration for those waves is longer. However, this effect is countered by the faster radial decay of the wind field in hurricanes with smaller $r_{m}$; the radial decay of the wind field can be approximately 

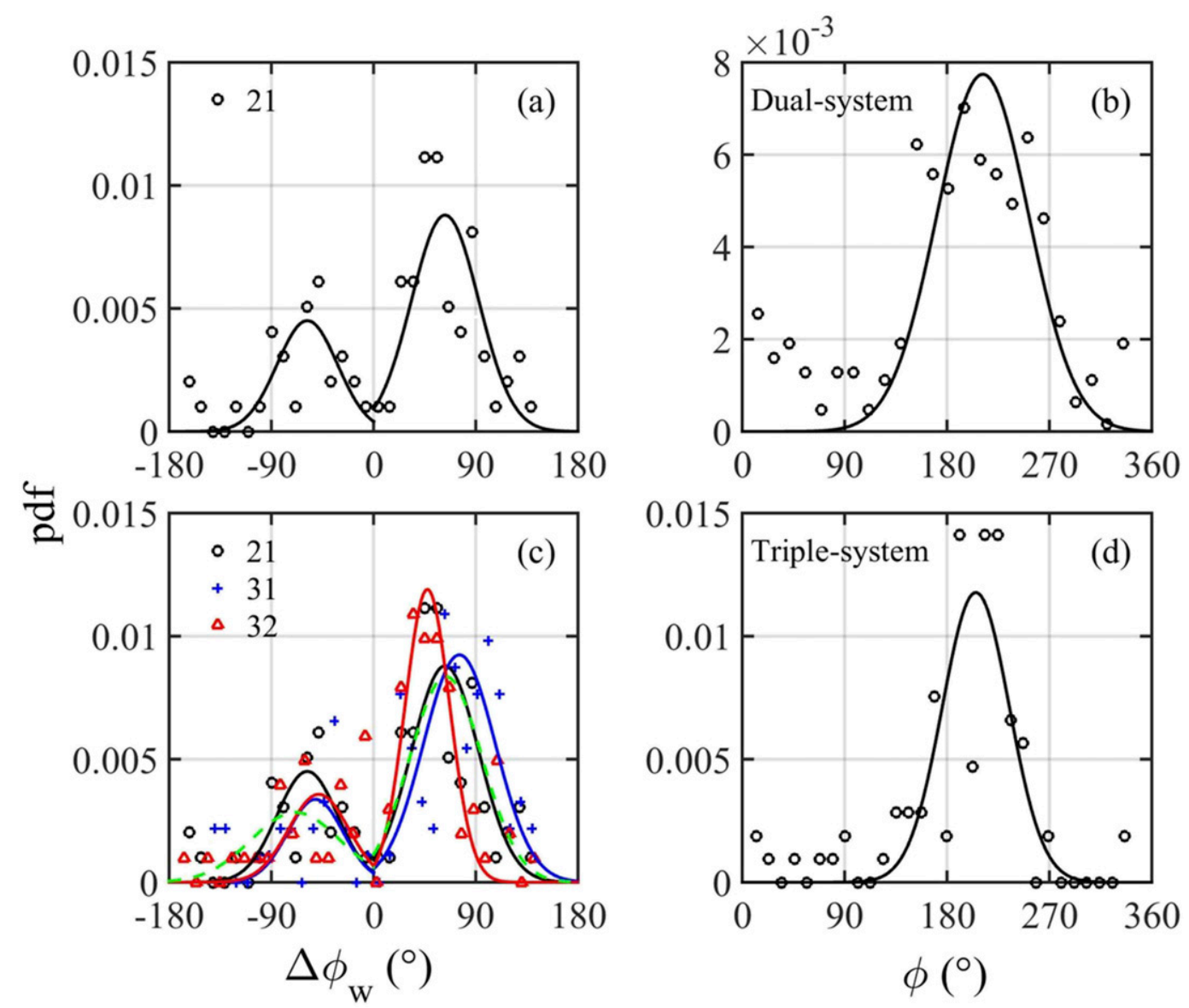

FIG. 10. The probability distributions of dual- and triple-system spectra: (a) dual-system directional difference between the secondary and the primary systems $\Delta \phi_{w 21}$; (b) dual-system azimuthal location of occurrence; (c) triplesystem directional differences of the secondary and tertiary systems from the primary system $\Delta \phi_{w 21}, \Delta \phi_{w 31}$, and the difference of the tertiary system from the secondary system $\Delta \phi_{w 32}$; and (d) triple-system azimuthal location of occurrence. The superimposed curves are Gaussian distributions computed with the parameters listed in Table 3. See text for more detail.

by $U_{10}(r) \approx U_{10 \max }\left(r / r_{m}\right)^{-0.5}$ for $r>r_{m}$ (e.g., Holland 1980; Holland et al. 2010).

The second region of special interest is in the left-back sector with $\phi_{w h}$ between about $90^{\circ}$ and $270^{\circ}$ : the generated waves propagate outward and away from the advancing wind field and thus receive decreased wind input in comparison to the other regions. The waves are much younger in terms of wave age and can be expected to be more active in wave breaking and air-sea exchanges.

\section{b. Multiple wave systems}

The propagation directions of spectra with multiple wave systems are investigated with the combined G1 and G2 datasets, which include 2425 spectra. Limiting the analysis to the region within $50 \leq r \leq 200 \mathrm{~km}$, there are totally 1650 spectra, for which $449(27.2 \%)$ have dual wave systems and 95 (5.8\%) have triple wave systems; only those multisystem spectra with the variance of the minor system(s) exceeding $20 \%$ variance of the primary system are accounted for. For the dual-system spectra, the average variance ratio of secondary to primary system $R_{v 21}$ (with one standard deviation) is $0.47 \pm 0.29$; the average wavenumber ratio $R_{k 21}=k_{p 2} / k_{p 1}$ is $1.34 \pm 0.64$. For the triple-system spectra, $R_{v 21}=0.74 \pm 0.54, R_{v 31}=$ $0.45 \pm 0.33, R_{v 32}=0.84 \pm 0.76, R_{k 21}=1.13 \pm 0.40$, $R_{k 31}=1.38 \pm 0.69$, and $R_{k 32}=1.28 \pm 0.68$.

Figures $10 \mathrm{a}$ and $10 \mathrm{~b}$ show the pdfs of the directional difference $\left(\Delta \phi_{w 21}\right)$ and the azimuthal location of occurrence of the dual-system spectra. Figures $10 \mathrm{c}$ and $10 \mathrm{~d}$ show the directional differences $\left(\Delta \phi_{w 21}, \Delta \phi_{w 31}\right.$, and $\left.\Delta \phi_{w 32}\right)$ and the azimuthal location of occurrence of the triple-system spectra.

All directional differences display bimodal distributions (Fig. 10, left column). They can be approximated 
TABLE 3. Parameters used in the Gaussian distribution curves shown in Fig. 10. Sum(pdf), Mean, and SD in the header are the integrated probability distribution, mean, and standard deviation, respectively. D21, D31, and D32 are $\Delta \phi_{w 21}, \Delta \phi_{w 31}$, and $\Delta \phi_{w 32}$, respectively; appended_2 and_3 represent dual- and triple-system spectra, respectively; appended $\mathrm{p}$ and $\mathrm{m}$ represent the angle range $\left(0^{\circ}\right.$ to $\left.150^{\circ}\right)$ and $\left(-150^{\circ}\right.$ to $\left.0^{\circ}\right)$, respectively; and for $\Delta \phi_{w 32}$, results calculated with the angle range $\left(0^{\circ}\right.$ to $\left.100^{\circ}\right)$ and $\left(-100^{\circ}\right.$ to $\left.0^{\circ}\right)$ are shown with appended px and mx, respectively. Phase_2 is the azimuthal location of the dual-system spectra, and Phase_3 is the azimuthal location of the triple-system spectra; the dual- and triplesystem statistics of the azimuthal location are computed for the range $135^{\circ} \leq \phi \leq 315^{\circ}$.

\begin{tabular}{lcrc}
\hline \multicolumn{1}{c}{ ID } & Sum(pdf) & Mean & SD \\
\hline D21_2p & 0.70 & 71.23 & 27.78 \\
D21_2m & 0.24 & -59.84 & 27.74 \\
Phase_2 & 0.78 & 211.47 & 40.34 \\
D21_3p & 0.65 & 62.99 & 29.61 \\
D21_3m & 0.30 & -58.35 & 26.56 \\
D31_3p & 0.74 & 75.59 & 31.81 \\
D31_3m & 0.21 & -51.12 & 24.29 \\
D32_3p & 0.67 & 63.73 & 31.89 \\
D32_3m & 0.28 & -68.76 & 39.21 \\
D32_3px & 0.58 & 47.42 & 19.57 \\
D32_3mx & 0.24 & -48.28 & 26.42 \\
Phase_3 & 0.87 & 205.35 & 29.42 \\
\hline
\end{tabular}

by two Gaussian curves fitted to the subpopulations with positive and negative difference angles. The integrated pdf, mean, and standard deviation for each subpopulation are listed in Table 3. To alleviate the distortion of statistics from the tail region, the calculation is carried out with the angle ranges $\left(0^{\circ}-150^{\circ}\right)$ and $\left(-150^{\circ}-0^{\circ}\right)$. For $\Delta \phi_{w 32}$, the tail distortion is rather severe and the calculations for $\left(0^{\circ}-100^{\circ}\right)$ and $\left(-100^{\circ}-0^{\circ}\right)$ are also performed. Overall, the minor systems are more likely propagating on the left-hand side of the primary system than on the right-hand side by a 3-to-1 ratio. The Gaussian curves computed with the parameters (mean and standard deviation) listed in Table 3 are superposed in Figs. 10a and 10c with smooth curves. For $\Delta \phi_{w 32}$, the solid red curves are the Gaussian distributions with parameters for $\left(0^{\circ}- \pm 100^{\circ}\right)$, and the green dashed curves are computed with the parameters for $\left(0^{\circ}- \pm 150^{\circ}\right)$; the former set of curves appear to give a better representation of the data distributions.

Most multiple-wave spectra occur in the region with $\phi$ from about $135^{\circ}$ to $305^{\circ}$ (Fig. 10, right column). The data can be approximately by Gaussian distributions as well; the statistics of the integrated probability, mean, and standard deviation calculated for the $\phi$ range from $135^{\circ}$ to $315^{\circ}$ are also listed in Table 3. For the dual system, 78\% occurs in this azimuth range with the phase mean and standard deviation of $211^{\circ} \pm 40^{\circ}$; for the triple system, the corresponding numbers are $87 \%$ and $205^{\circ} \pm 29^{\circ}$. The
Gaussian curves computed with those parameters are superposed in Figs. 10b and 10d with smooth curves.

\section{Summary}

In this paper, the $2 \mathrm{D}$ wavenumber spectra measured inside tropical cyclones in 11 hurricane reconnaissance and research missions during six hurricanes are analyzed. The azimuthal and radial variations of the wave propagation directions are presented graphically (Figs. 3-5) using two datasets with good spatial coverage (10 and 11 radial transects). The general spatial patterns are described in section 3. Among the major features are the dominance of monomodal propagation in the front half and front part of the left-back quarter, and the dominance of multimodal directional distributions in the back quarter and the rear part of the right quarter.

A parametric model of dominant wave propagation direction is then developed with the first four datasets listed in Table 1 and applied to all available datasets (section 4a). Overall comparison results are encouraging, showing consistent statistics in 9 of 11 datasets: the bias is less than $10^{\circ}$, the range of the linear regression slope is between 0.7 and 1.1, the rms difference is between 16 and $42^{\circ}$, and the correlation coefficient is better than 0.7 in 8 of the 9 datasets. The model performance may deteriorate considerably for hurricane data with close proximity to land (B26) and rapid change of hurricane translation direction or speed (H24).

The analysis results show that waves in the front-right quarter propagate into the advancing wind field, and they can grow higher and longer from the extended duration and fetch for air-sea exchanges. Waves in the rear-left quarter propagate away from the advancing wind field, and thus they are younger in terms of wave age. These features are consistent with observations obtained inside hurricanes with in situ buoys or remote sensing platforms (e.g., Wright et al. 2001; Walsh et al. 2002; Moon et al. 2003; Young 2006; Fan et al. 2009a,b; $\mathrm{Hu}$ and Chen 2011; Holthuijsen et al. 2012; EsquivelTrava et al. 2015; Fan and Rogers 2016; Collins et al. 2018), and the present analysis gives a more quantitative account.

Multisystem spectra locate mostly in the azimuthal sector $135^{\circ} \leq \phi \leq 315^{\circ}$ (Fig. 10, right column). The directional differences between secondary to primary, tertiary to primary, and tertiary to secondary all show bimodal distributions (Fig. 10, left column). Gaussian distribution curves using the parameters listed in Table 3 give a good approximation of the observed directional differences and the azimuthal locations of the multisystem spectra (section $4 b$ ). 

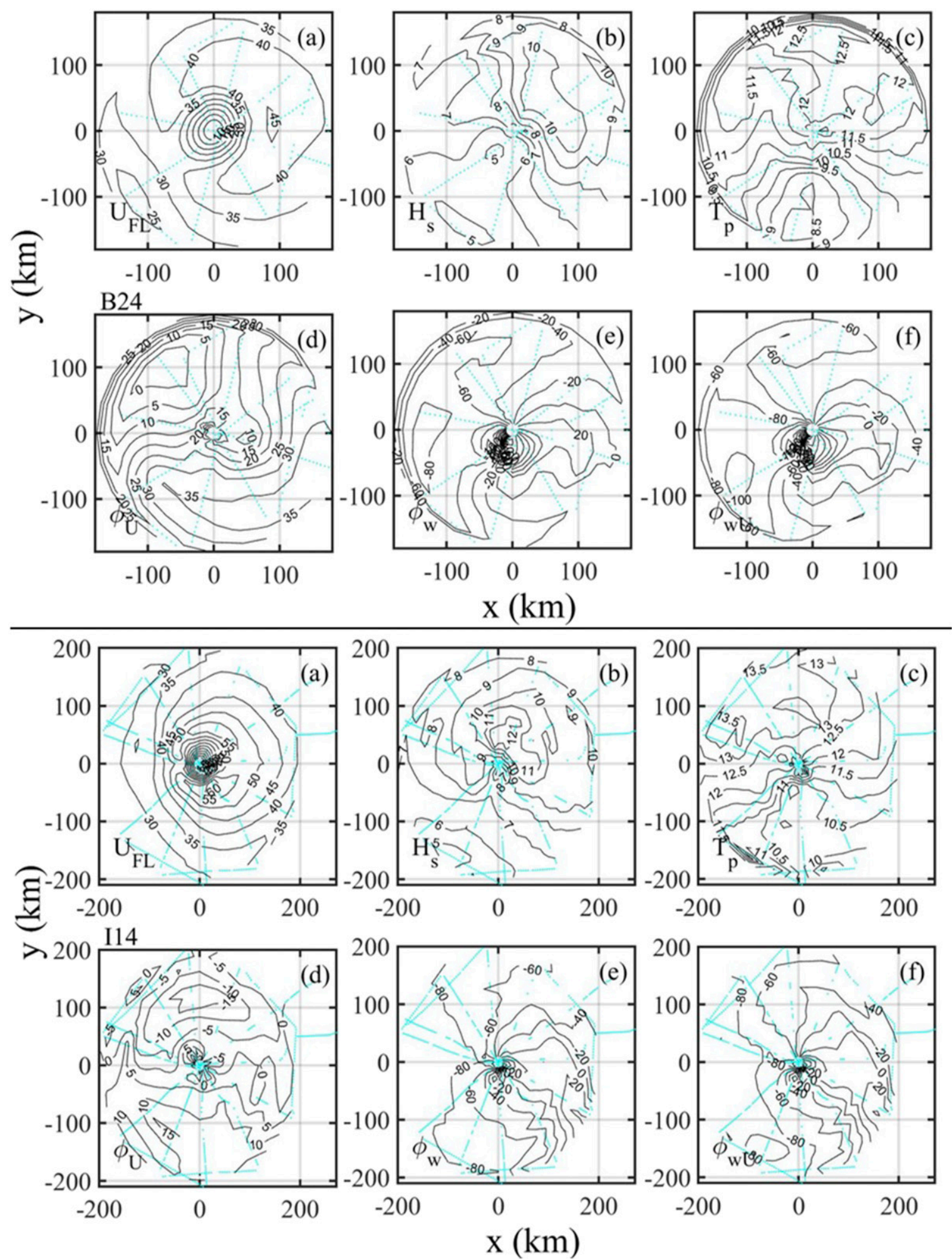

FIG. A1. 2D interpolated (a) $U_{\mathrm{FL}}$, (b) $H_{s}$, (c) $T_{p}$, (d) $\phi_{U}$, (e) $\phi_{w}$, and (f) $\phi_{w U}=\phi_{w}-\phi_{U}$. The B24 and I14 datasets are shown in the top and bottom halves of the figure, respectively. 
Acknowledgments. This work is sponsored by the Office of Naval Research (N0001416WX00044). We are grateful for the archive service of the HWIND legacy data maintained at http://www.rms.com/perils/hwind/ legacy-archive/. Datasets used in this analysis are given in the references cited. The processing codes and data segments can also be obtained by contacting the corresponding author. U.S. Naval Research Laboratory Publication Number JA/7260-18-0023.

\section{APPENDIX}

\section{D Interpolation of Aircraft Data}

The MATLAB function scatteredInterpolant performs interpolation on a $2 \mathrm{D}$ or $3 \mathrm{D}$ scattered dataset. For the 2D scattered data array $f_{i}=f\left(x_{i}, y_{i}\right)$, scatteredInterpolant returns a surface of the form $v=F(x, y)$. This surface always passes through the sample values at the point locations. The function $F$ is then used to calculate the value at any query point $\left(x_{q}, y_{q}\right)$ to produce an interpolated value $v_{q}$.

Because azimuthal variation is the primary feature of hurricane wind and wave data, the interpolation is applied in polar coordinates. To ensure interpolation for the full range of azimuth angle (e.g., $-180^{\circ}$ to $180^{\circ}$ ), the aircraft wind and wave data are repeated on both ends of the azimuthal range such that the azimuth angle covers $-360^{\circ}$ to $360^{\circ}$, that is, the scatteredInterpolant function is applied to the expanded dataset covering $-360^{\circ}$ to $360^{\circ}$, and the interpolation is performed for the range $-180^{\circ}$ to $180^{\circ}$.

In addition to wind field interpolation as shown in Fig. 2, the method can also be applied to the measured significant wave height $H_{s}$, dominant wave period $T_{p}$, as well as the subsequently derived quantities such as the wind direction $\phi_{U}$, dominant wave direction $\phi_{w}$, and the wind-wave directional difference $\phi_{w U}=\phi_{w}-\phi_{U}$. Figure A1 shows two sets of examples using the B24 (top two rows) and I14 (bottom two rows) datasets. In each panel, the aircraft ground tracks are superimposed in the background with light blue dots for reference.

The interpolated 2D fields clearly show the spatial patterns of the various wind and wave parameters, and they also effectively highlight the similarities and differences in the spatial patterns. For example, relative to the location of maximum wind speed, the locations of maximum $H_{s}$ and $T_{p}$ rotate $\mathrm{CCW}$ azimuthally and migrate outward radially: compare panels (a) to (c). The patterns of the wind and wave propagation directions are obviously different: compare panels (d) and (e). In terms of $\phi_{U}$ and $\phi_{w}$, the wind direction range is smaller than the wave direction range by a factor of about 4 , so the spatial pattern of the wind and wave directional difference is very close to the spatial pattern of the wave direction: compare panels (e) and (f).

The quality of the interpolation results depends on the data density as well as the data quality. For example, the edge effect in the B24 results, especially obvious in panels (b)-(f), is the consequence of insufficient coverage in the outer range. The interpolation is performed for $r$ between 0 and $200 \mathrm{~km}$, but the measurements are mostly within about $r<185 \mathrm{~km}$ in B24 (see left column of Fig. 2).

\section{REFERENCES}

Black, P. G., and Coauthors, 2007: Air-sea exchange in hurricanes: Synthesis of observations from the Coupled Boundary Layer Air-Sea Transfer Experiment. Bull. Amer. Meteor. Soc., 88, 357-374, https://doi.org/10.1175/BAMS-88-3-357.

Collins, C. O., H. Potter, B. Lund, H. Tamura, and H. C. Graber, 2018: Directional wave spectra observed during intense tropical cyclones. J. Geophys. Res. Oceans, 123, 773-793, https:// doi.org/10.1002/2017JC012943.

Dunion, J. P., and C. S. Velden, 2002: Application of surfaceadjusted GOES low-level cloud-drift winds in the environment of Atlantic tropical cyclones. Part II: Integration into surface wind analysis. Mon. Wea. Rev., 130,1347-1355, https:// doi.org/10.1175/1520-0493(2002)130<1347:AOSAGL>2.0.CO;2.

— S. H. Houston, C. S. Velden, and M. D. Powell, 2002: Application of surface-adjusted GOES low-level cloud-drift winds in the environment of Atlantic tropical cyclones. Part I: Methodology and validation. Mon. Wea. Rev., 130,1333-1346, https://doi.org/10.1175/1520-0493(2002)130<1333:AOSAGL> 2.0.CO;2.

, C. W. Landsea, S. H. Houston, and M. D. Powell, 2003: A reanalysis of the surface winds for Hurricane Donna of 1960. Mon. Wea. Rev., 131, 1992-2011, https://doi.org/10.1175/15200493(2003)131<1992:AROTSW>2.0.CO;2.

Esquivel-Trava, B., F. J. Ocampo-Torres, and P. Osuna, 2015: Spatial structure of directional wave spectra in hurricanes. Ocean Dyn., 65, 65-76, https://doi.org/10.1007/s10236-0140791-9.

Fan, Y., and W. E. Rogers, 2016: Drag coefficient comparisons between observed and model simulated directional wave spectra under hurricane conditions. Ocean Modell., 102, 1-13, https://doi.org/10.1016/j.ocemod.2016.04.004.

_ I. Ginis, and T. Hara, 2009a: The effect of wind-wave-current interaction on air-sea momentum fluxes and ocean response in tropical cyclones. J. Phys. Oceanogr., 39, 2097-2116, https:// doi.org/10.1175/2008JPO4066.1.

, C. W. Wright, and E. J. Walsh, 2009b: Numerical simulations and observations of surface wave fields under an extreme tropical cyclone. J. Phys. Oceanogr., 39, 2097-2116, https://doi.org/10.1175/2009JPO4224.1.

Franklin, J. L., M. L. Black, and K. Valde, 2003: GPS dropwindsonde wind profiles in hurricanes and their operational implications. Wea. Forecasting, 18, 32-44, https://doi.org/10.1175/ 1520-0434(2003)018<0032:GDWPIH >2.0.CO;2.

Holland, G. J., 1980: An analytic model of the wind and pressure profiles in hurricanes. Mon. Wea. Rev., 108, 1212-1218, https://doi.org/10.1175/1520-0493(1980)108<1212:AAMOTW > 2.0.CO;2. 
J. I. Belanger, and A. Fritz, 2010: A revised model for radial profiles of hurricane winds. Mon. Wea. Rev., 138, 4393-4401, https://doi.org/10.1175/2010MWR3317.1.

Holthuijsen, L. H., M. D. Powell, and J. D. Pietrzak, 2012: Wind and waves in extreme hurricanes. J. Geophys. Res., 117, C09003, https://doi.org/10.1029/2012JC007983.

$\mathrm{Hu}, \mathrm{K}$., and Q. Chen, 2011: Directional spectra of hurricanegenerated waves in the Gulf of Mexico. Geophys. Res. Lett., 38, L19608, https://doi.org/10.1029/2011GL049145.

Hwang, P. A., 2016: Fetch- and duration-limited nature of surface wave growth inside tropical cyclones: With applications to airsea exchange and remote sensing. J. Phys. Oceanogr., 46, 4156, https://doi.org/10.1175/JPO-D-15-0173.1.

_ , and E. J. Walsh, 2016: Azimuthal and radial variation of windgenerated surface waves inside tropical cyclones. J. Phys. Oceanogr., 46, 2605-2621, https://doi.org/10.1175/JPO-D-16-0051.1.

_ cyclone wind fields estimated from simultaneous wind and wave measurements: Surface wave and air-sea exchange computation. J. Phys. Oceanogr., 47, 447-470, https://doi.org/ 10.1175/JPO-D-16-0180.1.

_- and E. J. Walsh, 2018: Estimating maximum significant wave height and dominant wave period inside tropical cyclones. Wea. Forecasting, https://doi.org/10.1175/WAF-D-17-0186.1, in press.

— , Y. Fan, F. J. Ocampo-Torres, and H. García-Nava, 2017: Ocean surface wave spectra inside tropical cyclones. J. Phys. Oceanogr., 47, 2393-2417, https://doi.org/10.1175/JPO-D-17-0066.1.

Moon, I.-J., I. Ginis, T. Hara, H. L. Tolman, C. W. Wright, and E. J. Walsh, 2003: Numerical simulation of sea surface directional wave spectra under hurricane wind forcing. J. Phys. Oceanogr., 33, 1680-1706, https://doi.org/10.1175/2410.1.

Powell, M. D., 1980: Evaluations of diagnostic marine boundary layer models applied to hurricanes. Mon. Wea. Rev., 108, 757-766, https://doi.org/10.1175/1520-0493(1980)108<0757: EODMBL $>2.0 . \mathrm{CO} ; 2$.

_ 1982: The transition of the hurricane Frederic boundary-layer wind field from the open Gulf of Mexico to landfall. Mon.
Wea. Rev., 110, 1912-1932, https://doi.org/10.1175/1520-0493 (1982) $110<1912:$ TTOTHF $>2.0 . C O ; 2$.

— , and S. H. Houston, 1998: Surface wind fields of 1995 Hurricanes Erin, Opal, Luis, Marilyn, and Roxanne at landfall. Mon. Wea. Rev., 126, 1259-1273, https://doi.org/10.1175/15200493(1998)126<1259:SWFOHE $>2.0$.CO;2

,-- , and T. A. Reinhold, 1996: Hurricane Andrew's landfall in south Florida. Part I: Standardizing measurements for documentation of surface wind fields. Wea. Forecasting, 11, 304-328, https://doi.org/10.1175/1520-0434(1996)011<0304: HALISF $>2.0 . \mathrm{CO} ; 2$.

Uhlhorn, E. W., and P. G. Black, 2003: Verification of remotely sensed sea surface winds in hurricanes. J. Atmos. Oceanic Technol., 20, 99-116, https://doi.org/10.1175/1520-0426(2003) $020<0099$ :VORSSS $>2.0$.CO;2.

, - J. L. Franklin, M. Goodberlet, J. Carswell, and A. S. Goldstein, 2007: Hurricane surface wind measurements from an operational stepped frequency microwave radiometer. Mon. Wea. Rev., 135, 3070-3085, https://doi.org/10.1175/MWR3454.1.

Walsh, E. J., D. W. Hancock, D. E. Hines, R. N. Swift, and J. F. Scott, 1985: Directional wave spectra measured with the surface contour radar. J. Phys. Oceanogr., 15, 566-592, https://doi.org/ 10.1175/1520-0485(1985)015<0566:DWSMWT>2.0.CO;2.

,,,---- , and -1989 : An observation of the directional wave spectrum evolution from shoreline to fully developed. J. Phys. Oceanogr., 19, 670-690, https://doi.org/ 10.1175/1520-0485(1989)019<0670:AOOTDW >2.0.CO;2.

— , and Coauthors, 2002: Hurricane directional wave spectrum spatial variation at landfall. J. Phys. Oceanogr., 32, 1667-1684, https://doi.org/10.1175/1520-0485(2002)032<1667:HDWSSV> 2.0.CO;2.

Wright, C. W., and Coauthors, 2001: Hurricane directional wave spectrum spatial variation in the open ocean. J. Phys. Oceanogr., 31, 2472-2488, https://doi.org/10.1175/1520-0485(2001) $031<2472:$ HDWSSV $>2.0 . \mathrm{CO} ; 2$.

Young, I. R., 2006: Directional spectra of hurricane wind waves. J. Geophys. Res., 111, C08020, https://doi.org/10.1029/ 2006JC003540. 